\title{
Characterization of Temperature Induced Phase Transitions in the Five Polymorphic Forms of Sulfathia-zole by Terahertz Pulsed Spectroscopy and Differential Scanning Calorimetry
}

Zeitler, J. Axel; Newnham, David A.; Taday, Philip F.; Threlfall, Terry L.; Lancaster, Robert W.; Berg, Rolf W.; Strachan, Clare J.; Pepper, Michael; Gordon, Keith C.; Rades, Thomas

Published in:

Journal of Pharmaceutical Sciences

Link to article, DOI:

10.1002/jps.20719

Publication date:

2006

Link back to DTU Orbit

Citation (APA):

Zeitler, J. A., Newnham, D. A., Taday, P. F., Threlfall, T. L., Lancaster, R. W., Berg, R. W., Strachan, C. J., Pepper, M., Gordon, K. C., \& Rades, T. (2006). Characterization of Temperature Induced Phase Transitions in the Five Polymorphic Forms of Sulfathia-zole by Terahertz Pulsed Spectroscopy and Differential Scanning Calorimetry. Journal of Pharmaceutical Sciences, 95(11), 2486-2498. https://doi.org/10.1002/jps.20719

\section{General rights}

Copyright and moral rights for the publications made accessible in the public portal are retained by the authors and/or other copyright owners and it is a condition of accessing publications that users recognise and abide by the legal requirements associated with these rights.

- Users may download and print one copy of any publication from the public portal for the purpose of private study or research.

- You may not further distribute the material or use it for any profit-making activity or commercial gain

- You may freely distribute the URL identifying the publication in the public portal 


\title{
Characterization of Temperature Induced Phase
}

\section{Transitions in the Five Polymorphic Forms of}

\section{Sulfathiazole by Terahertz Pulsed Spectroscopy and Differential Scanning Calorimetry}

\author{
J. Axel Zeitler ${ }^{1,2,3}$, David A. Newnham ${ }^{3}$, Philip F. Taday ${ }^{3,}$, Terry L. Threlfall ${ }^{4}$, Robert \\ W. Lancaster ${ }^{5}$, Rolf W. Berg ${ }^{6}$, Clare J. Strachan ${ }^{1, \dagger}$, Michael Pepper ${ }^{2,3}$, Keith C. \\ Gordon $^{7}$, Thomas Rades ${ }^{1}$ \\ 1 School of Pharmacy, University of Otago, P.O. Box 56, Dunedin, New Zealand \\ 2 Cavendish Laboratory, University of Cambridge, Madingley Road, Cambridge, CB3 OHE, United Kingdom \\ 3 TeraView Limited, Platinum Building, St John's Innovation Park, Cambridge, CB4 0WS, United Kingdom \\ 4 School of Chemistry, University of Southampton, Southampton, SO17 1BJ, United Kingdom \\ 5 Department of Chemistry, University College London, Gordon Street, London, WC1H 0AJ, United Kingdom \\ ${ }^{6}$ Department of Chemistry, DTU (Technical University of Denmark), Kemitorvet, Building 207, DK-2800 \\ Lyngby, Denmark \\ 7 Department of Chemistry, University of Otago, P.O. Box 56, Dunedin, New Zealand \\ * Corresponding author. Tel.: +44 1223 435388; fax: +44 1223435382. \\ E-mail address: philip.taday@teraview.com (P.F. Taday). \\ ${ }^{\dagger}$ Present address: Pharmaceutical Technology Division, Faculty of Pharmacy, 00014 University of Helsinki, P.O. \\ Box 56, Helsinki. Finland
}

\section{Abstract}

The far-infrared properties of all five described polymorphic forms of the drug sulfathiazole have been studied by terahertz pulsed spectroscopy and low frequency Raman spectroscopy. The spectra of the different polymorphs are distinctly different. Terahertz pulsed spectroscopy proves to be a rapid complementary alternative to other physical characterisation techniques reported in the literature for distinguishing 
between the five forms. Variable temperature measurements ( $293 \mathrm{~K}$ to $473 \mathrm{~K}$ ) of all polymorphic forms have been performed. The phase transitions observed have been related to thermal analysis data. Form I is the stable high temperature form of sulfathiazole with a melting point of about $475 \mathrm{~K}$. Form II melts at around $470 \mathrm{~K}$ and recrystallizes at higher temperatures to form I. Forms III to V all convert to form I via a solid-solid phase transition at temperatures below $450 \mathrm{~K}$. The phase transitions can be monitored by terahertz pulsed spectroscopy. Common polymorphic impurities of the samples can be detected in the room temperature spectra and their effect on the phase transition behaviour can be studied.

Key words: Terahertz spectroscopy; low frequency Raman spectroscopy; farinfrared; sulfathiazole; polymorphism; phase transitions; DSC; high speed DSC; physical characterization; lattice vibrations

\section{Introduction}

The antibiotic drug sulfathiazole and its crystalline properties have been extensively described and studied since the 1940s. ${ }^{1}$ Even though marked as the classic polymorphic system by Burger and Dialer over twenty years ago, ${ }^{2}$ the structure of a fifth polymorph was only determined quite recently. ${ }^{3-5}$ A number of different techniques have been used for the physical characterisation of the polymorphic forms including thermal analysis, vibrational spectroscopy, solid-state NMR, and X-ray methods. ${ }^{1,2,6,7}$ Despite the thorough research into this system some properties of sulfathiazole remain a challenge to understand. ${ }^{8-10}$ The nomenclature of the polymorphs is not consistent in the literature and may lead to confusion as different systems in use 
today all use Roman numerals. In this paper we use the convention clarified by Apperley et al. ${ }^{1}$

Low frequency radiation in the far-infrared region of the electromagnetic spectrum, $2 \mathrm{~cm}^{-1}-133 \mathrm{~cm}^{-1}(60 \mathrm{GHz}-4 \mathrm{THz})$, is generally referred to as terahertz radiation. The spectral range covered overlaps the millimetre and microwave part of the spectrum to the low frequency end and the mid-infrared region to the higher frequency region. Terahertz radiation excites low energy vibrations associated with coherent, delocalized movements of molecules or molecular fragments rather than the individual atoms within the molecules. ${ }^{11}$ Recent advances in ultra-fast laser systems and the development of GaAs optical switches allowed the emission and detection of coherent broadband pulsed terahertz radiation at room temperature. ${ }^{12}$ Spectroscopy in the farinfrared has thus become much more attractive and more widely deployed. Consequently terahertz pulsed spectroscopy (TPS) has been demonstrated to be a powerful tool for the measurement of low-frequency vibrational modes. ${ }^{13-15}$ Optical gating techniques are used for detection of the pulsed radiation, recording the transient electric field rather than just the intensity of the terahertz radiation. This allows the direct determination of amplitude and phase in TPS measurements. Using this information absorption coefficients and spectral refractive indices can be calculated without the need to invoke the Kramers-Kronig dispersion relationship. ${ }^{13}$ Taking account of these advantages, TPS is being increasingly used to study low-frequency vibrational modes for a wide variety of samples including chemical, biological, pharmaceutical, and security-related materials such as explosives. ${ }^{16-24}$ 
Since sulfathiazole is reported in the literature as a classic example of a drug with five polymorphs and the differentiation between the forms is far from trivial, we have chosen it as a suitable system to test the capabilities of TPS.

\section{Experimental}

2.1 Materials - The polymorphic forms of sulfathiazole studied in this paper were prepared as described by Apperley et al. ${ }^{1}$ Poly(tetrafluoroethylene) (PTFE) and polyethylene (PE) were obtained from Sigma-Aldrich (Poole, UK).

2.2 Sample preparation - For the qualitative comparison of the different polymorphic forms at room temperature, $20 \mathrm{mg}$ of the sample was gently ground and mixed with $400 \mathrm{mg}$ PE. For the reference $400 \mathrm{mg}$ PE was used. The variable temperature measurements were performed with a sample containing $15-20 \mathrm{mg}$ of each sulfathiazole polymorph physically mixed with $550 \mathrm{mg}$ PTFE. Using a die press (Specac, Orpington, UK) all mixtures and references were compressed with 1 ton load for $3 \mathrm{~min}$ into a pellet of $13 \mathrm{~mm}$ diameter.

2.3 Qualitative terahertz pulsed spectroscopy - Using a $13 \mathrm{~mm}$ sample holder spectra of the sample and reference pellets were recorded on a TPS spectra1000 spectrometer (TeraView, Cambridge, UK) at an instrument resolution of $1 \mathrm{~cm}^{-1}$ over the range of $2 \mathrm{~cm}^{-1}$ to $95 \mathrm{~cm}^{-1}$. Throughout the measurements, the sample chamber was purged with dry nitrogen. Sample and reference spectra (1800 scans co-added) were recorded, resulting in an acquisition time of one minute per spectrum.

2.4 Temperature dependent terahertz pulsed spectroscopy - The sample pellets were held in a brass ring with a circular aperture of $8 \mathrm{~mm}$ and inserted into a 
heatable transmission cell (Specac, Orpington, UK) without windows. The sample temperature was controlled by a 3000 series high stability temperature controller (Specac, Orpington, UK). Temperature was calibrated using compounds of known melting point. The sample pellet temperature was increased at a rate of approximately $2 \mathrm{~K} \mathrm{~min}^{-1}$. Terahertz spectra were recorded with a TPS spectra1000 spectrometer (TeraView, Cambridge, UK) using an instrument resolution of $1.5 \mathrm{~cm}^{-1}$ over the range of $2 \mathrm{~cm}^{-1}$ to $95 \mathrm{~cm}^{-1}$ as previously described. ${ }^{23}$ To reduce the contribution of atmospheric water vapour the sample chamber was purged with dry nitrogen throughout the experiment. Spectra were recorded by co-adding 1800 scans (one minute total). Each sample spectrum was referenced against a spectrum of a pellet of $550 \mathrm{mg}$ PTFE, with nitrogen purging.

2.5 Spectral processing - Sample and reference spectra were calculated by a fast Fourier transformation of the time-domain waveform. Spectra were zerofilled at a factor of 2 and apodized using the 3-term Blackman-Harris procedure. Absorbance spectra were calculated from the sample and reference spectra. To compensate for scattering effects at higher frequencies the spectra of the qualitative measurements were baseline corrected using a simple power function. For better comparability the spectra were normalised. The spectra of the temperature dependent measurements were used as recorded and no processing was performed. Measurements and spectrum processing were carried out using TPI Spectra 1.9.8 (TeraView, Cambridge, UK).

2.7 Low frequency Raman spectroscopy - Raman spectra were measured with a DILOR-XY $800 \mathrm{~mm}$ focal length multichannel spectrometer with microscope entrance, horizontal Ar-ion laser excitation $(514.5 \mathrm{~nm}, 300 \mathrm{~mW}$, vertically polarized), and liquid $\mathrm{N}_{2}$-cooled CCD light detection. Rayleigh scattered light was filtered off with a double 
pre-monochromator (slits $200 \mu \mathrm{m}$ ). The Raman spectral resolution was about $4 \mathrm{~cm}^{-1}$ and the precision about $1 \mathrm{~cm}^{-1}$, obtained by calibration with Raman lines from crystalline $\mathrm{S}_{8}{ }^{25}$ Samples were measured without further sample preparation.

2.7 Heat flux differential scanning calorimetry (DSC) - DSC was performed using a TA Q1000 (TA Instruments, New Castle, USA) differential scanning calorimeter based on the Boersma design (heat flux). The instrument was calibrated using indium standards. Between $1 \mathrm{mg}$ and $2 \mathrm{mg}$ per sample of the respective sulfathiazole form was weighed into aluminium pans and sealed. Under helium gas purge the samples were equilibrated at $293 \mathrm{~K}$ and then heated up to $483 \mathrm{~K}$ at ramp rates of $2 \mathrm{~K} \mathrm{~min}^{-1}, 10 \mathrm{~K} \mathrm{~min}^{-1}$ and $150 \mathrm{~K} \mathrm{~min}^{-1}$.

2.8 Power compensation high speed DSC - A Diamond DSC (PerkinElmer, UK) was used for recording the DSC traces of the different polymorphs at fast heating rates. The instrument was cooled by an Intracooler II (PerkinElmer, UK). Calibration was performed using indium standards at the respective heating rate. For each sample $0.2 \mathrm{mg}$ to $1.4 \mathrm{mg}$ of the sample material was weighed into aluminium pans. Helium was used as purge gas. The samples were equilibrated at $233 \mathrm{~K}$ and then heated to $523 \mathrm{~K}$ at ramp rates of $300 \mathrm{~K} \mathrm{~min}^{-1}$ and $500 \mathrm{~K} \mathrm{~min}^{-1}$. A baseline of an empty pan was recorded for each heating rate and subtracted from the sample DSC trace.

2.9 Hot stage polarising light microscopy - A Nikon Optiphot phase contrast light microscope equipped with polarizer and analyser and a hot stage FP82HT with a central processor FP90 (Mettler Toledo, USA) were used. The heating range was 298 $483 \mathrm{~K}$ and the heating rate was $10 \mathrm{~K} \mathrm{~min}^{-1}$. 


\section{Results and Discussion}

3.1 Far infrared spectra of sulfathiazole polymorphic forms - The terahertz absorption spectra of the five different polymorphic forms described are shown in Figure 2A. Table 1 lists the positions of the peaks of all polymorphic forms in the terahertz region. All forms show distinct spectral features in the range between $2 \mathrm{~cm}^{-1}$ and $95 \mathrm{~cm}^{-1}$. They can be easily distinguished from one another by their terahertz absorption spectra. In contrast to mid-IR that is lacking specificity in the analysis of this system, this makes TPS a very valuable tool to study this polymorphic system in addition to established techniques like X-ray powder diffraction (XRPD) and solid-state NMR. ${ }^{1}$ The corresponding low-frequency Raman spectra are shown in Figure 2B. Figure 3 shows that above $150 \mathrm{~cm}^{-1}$ the spectral features become less pronounced, less frequent and more isolated. The majority of the phonon modes are expected below $150 \mathrm{~cm}^{-1} .{ }^{11}$ Using the Raman technique all five polymorphic forms can also be distinguished from one another definitively. However, the effort to acquire the spectra is more significant using the Raman setup as the maintenance and alignment of these systems is more demanding and the acquisition times are much longer. The recording of Raman spectra so close to the laser line also requires a triple monochromator Raman system. Choosing the optimal laser excitation wavelength and the right balance between acquisition time and laser excitation power requires much expertise. For more complex samples, that may include excipients, the problem of fluorescence emission furthermore can swamp the Raman signal. An advantage of the Raman method is however the possibility for in-situ measurements on virgin samples under the microscope. 
Compared to the spectra of the other four forms, the spectrum of polymorph I is relatively featureless at room temperature. The IR active modes show one major peak at $65 \mathrm{~cm}^{-1}$ with all the other spectral features appearing very weak. The dominance of this peak may be due to the fact that form I is composed of two interlocked lattices known to exhibit a very high anisotropic lattice expansion. ${ }^{1}$ This might lead to a very strong phonon mode. The spectrum of the Raman active modes is also the most featureless for form I compared to the other forms. The peak positions for polymorph III lie between those of polymorphs IV and V. The terahertz spectral features of form III are more similar to those of form IV than form V. The crystal structures of all of the five polymorphs investigated have been determined. ${ }^{1}$ Polymorph IV consists of dimers joined to form chains in one dimension linked to identical chains orthogonally building a layer which will be referred to here as an A layer. So polymorph IV consists of stacks of layers AAAAAA. Polymorph V has identical chains but joined differently to give B layers, so the structure is BBBBBB. Polymorph III contains alternate A and B layers and has a structure $\mathrm{ABABAB}$. The consequence of this is that in all properties IV and $\mathrm{V}$ are very similar whilst III is either the sum of IV and V or intermediate. In near infrared, mid infrared, Raman and NMR regimes the spectrum of form III is virtually the sum of that of IV and V. In X-ray powder diffraction (XRPD) and far-Raman spectra, the peaks of III lie between those of IV and V so specimen of III can be distinguished from a 50/50 mixture of IV and V. However, due to the closeness of the peaks and overlap from the peaks of I or II if present, definite distinction relies on the use of more than one technique. Furthermore there are other polymorphs, presumably of structures such as $\mathrm{AABBAA}$ or $\mathrm{AABAAB}$, which occasionally produce further interference. A NMR spectrum of one of these is described and depicted by Apperley et $a l .{ }^{1}$ By terahertz spectroscopy, the peaks of III lie between these of IV and V and are 
well separated, thus allowing in principle distinction between these polymorphs and quantification of mixtures. As form I and II can also be distinguished very well from the forms III, IV and V all forms can be discriminated from one another by examination of their terahertz spectra.

In XRPD preferred orientation effects have a very strong impact on the nature of the diffraction pattern. The crystals of sulfathiazole form III, IV and V can have distinct plate-like morphology. In this case it is very difficult to ensure random orientation of the particles which is a prerequisite to record powder patterns representing the true lattice structure of the sample. As a result, the diffractogram is distorted with intensities of certain lines greatly emphasised against the other XRPD lines. Furthermore, the positions of the most intense and characteristic bands are very close together for the different forms of sulfathiazole and care must be taken to avoid misinterpretations. Misinterpretations of the diffraction patterns can, and frequently do, occur. Particularly, the conclusion is often drawn that a sample is polymorphically pure by XRPD when it is a gross mixture.

Solid-state NMR spectra of the different forms of sulfathiazole are recorded using cross polarized magic angle spinning. All polymorphs can be distinguished from one another and a high degree of chemical information relating to the molecule in the crystal lattice can be obtained. ${ }^{1}$ However, it takes several hours and a substantial amount of sample to record a good quality spectrum of a single sample with sufficient signal to noise ratio. Using DSC it is not possible to distinguish between these forms as their melting endotherms are all in the same temperature range as discussed below. 
Even though TPS is a good tool for the fast and reliable differentiation of polymorphic forms more understanding of the interpretive assignment of the spectral features in the terahertz spectra would be beneficial. An understanding of the spectra may facilitate studies of solid state reaction mechanisms. The assignment of specific features in this region of the electromagnetic spectrum is still in its infancy. So far few studies have been published in which density functional theory (DFT) calculations have been used to interpret terahertz spectra. We have studied the intramolecular low frequency predictions of DFT calculations of carbamazepine in its single molecule as well as in the dimer structure. ${ }^{24}$ Even though it is possible that most modes in the terahertz spectra are caused or influenced by low energy intramolecular vibrations it is apparent that it is not sufficient to focus on single molecule structures to explain all the features observed. Using a more advanced computational approach we were able to calculate the lattice dynamics for the different polymorphs of carbamazepine rather than the isolated dimer structure in order to understand the low frequency phonon modes in more depth. ${ }^{11}$ Tentative assignments of the measured spectral features to certain lattice vibrations of two polymorphs were made leading to a better understanding of the terahertz spectra. These calculations can be regarded as a first important step towards a rational and full understanding of terahertz spectra. However, further work is necessary to see whether the results obtained in the case of a rigid molecular structure translate to less rigid molecular crystals, and at which part of the spectrum the effects of intramolecular vibration begin to dominate.

The spectra presented for polymorph II and IV in Figure 2 and Figure 3 are unlikely to be of $100 \%$ polymorphic purity (see discussion below). However the 
samples analysed are of the highest polymorphic purity that could be obtained and it was possible to unambiguously identify impurities in them.

3.2 Thermal analysis - By DSC, the different polymorphic forms III to $\mathrm{V}$ are found to convert to form I at varying temperatures between $420 \mathrm{~K}$ and $450 \mathrm{~K}$ via a solid-solid transformation. ${ }^{6,7}$ Further heating leads to melting of form I at around $474 \mathrm{~K}$ (Figure 4A-E). The conversion temperature of forms III to $\mathrm{V}$ depends on the heating rate. The endotherms of the conversion process are recorded in about the same temperature range for all the different forms (Table 2). Form II melts at $469 \mathrm{~K}$. For slower heating rates this melting endotherm is followed by an immediate recrystallization exotherm as the sample crystallizes to form I.

Samples of form II are quite unstable and if not dried in the appropriate conditions straight after their crystallization, they can be easily contaminated with form III, IV or $\mathrm{V}$ by immediate conversion. In this case a solid-solid conversion to form I between $420 \mathrm{~K}$ and $450 \mathrm{~K}$ of the contaminant can be observed in addition to the melt of form II at $469 \mathrm{~K}$. Even though great care was taken in order to analyse pure polymorphs of all forms it was not possible to record a pure specimen of form II due to its instability. The polymorphic purity of form II was confirmed by FT-IR straight after crystallization but partial transformation occurred during transport to the DSC measurements. As it was intended to compare the DSC measurements with the terahertz spectra, the DSC traces were recorded prior to the terahertz measurements at the same site. To demonstrate the instability of form II the DSC trace of one sample was recorded immediately after crystallization at a heating rate of $300 \mathrm{~K} \mathrm{~min}^{-1}$ (Figure 4D compared to E) 
It is difficult, if not impossible, to distinguish the different polymorphic forms by thermal analysis alone. In DSC, measurements of polycrystalline samples represent the melting behaviour of the sample bulk. The conversion to form I is a defect driven process, ${ }^{1,26}$ so the conversion endotherm to form I is more sample dependent than characteristic for its polymorphic form. Even though the thermodynamic transition point for this process is theoretically at $368 \mathrm{~K}$, no conversion is reported in the literature for temperatures below $400 \mathrm{~K} .{ }^{27}$ It was demonstrated by DSC measurements of sulfathiazole form III single crystals at low heating rates that the conversion temperature for this specific form is distributed over the range between $421 \mathrm{~K}$ and $433 \mathrm{~K}$ depending on the individual sample crystal. ${ }^{28}$ In general the endotherms have lower onset temperatures at slower heating rates. This is due to the slow kinetics of transformation. The true transition temperature for the conversion to form I is known to be about $368 \mathrm{~K}$ to $389 \mathrm{~K}$. Using high heating rates in DSC reveals additional information. At slow heating rates annealing processes can occur and some of the defect structures can be eliminated. This effect is not observed at high heating rates as the annealing processes are slow. As a result, the conversion endotherms recorded at slow heating rates have a lower onset temperature compared with the onset temperature recorded at high heating rates.

Increasing the heating rate also leads to a partial inhibition of the recrystallization of the lower melting polymorph revealing the true melting point of the polymorphic form. This effect has been recently demonstrated by heating carbamazepine at rates of up to $250 \mathrm{~K} \mathrm{~min}^{-1}{ }^{29}$ The kinetics of solid-solid transformations is often slow and so the transition can be delayed or prevented. This also explains the shift in transition temperatures towards higher temperatures for the forms II to IV recorded at faster 
heating rates. A heating rate of $150 \mathrm{~K} \mathrm{~min}^{-1}$ is the upper limit for the heat flux instrument setup used for this experiment and the melting enthalpies should not be considered accurate for these measurements. This is due to the fact that in this setup both reference and sample are heated by the same oven and the temperature difference between the two is recorded during heating. As the temperature of a rather big compartment is controlled the lag time of the system is significant and restricts the heating rate. However, the values are reproducible and the aim of the experiment at $150 \mathrm{~K} \mathrm{~min}^{-1}$ is to investigate the qualitative effects of the heating rate on the conversion endotherm observed at lower temperatures rather than giving accurate enthalpies for the melting events (Figure 4C). To examine the effects at even higher heating rates experiments were performed using a power compensation setup DSC (Figure 4D-E). This setup allows accurate measurements of the thermal properties at heating rates up to $500 \mathrm{~K} \mathrm{~min}^{-1}$ as sample and reference are heated by two separate smaller ovens. Rather than measuring the temperature difference between sample and reference during heating the temperature of both is kept equal throughout the experiment by adjusting the heating power of the individual heating elements. These experiments were very helpful to confirm the findings form the heat flux setup and to make accurate measurements of the melting enthalpy. As Table 2 confirms using the heat flux DSC setup it was not possible to obtain accurate enthalpies at heating rates of $150 \mathrm{~K} \mathrm{~min}^{-1}$.

Depending on the particle size distribution in the sample more than one endotherm may be observed in the DSC trace of form III, IV or V at temperatures below $450 \mathrm{~K}$. Under a polarizing light microscope it can be observed that some fractions of the sample tend to convert to form I via melt and recrystallization whereas bigger particles convert via a solid-solid transformation process (Figure 5). This type of 
mixed behaviour is confirmed by DSC of a different sample of form IV. A broad endotherm of the solid-solid conversion is recorded. The onset temperature is heating rate dependent between $425 \mathrm{~K}$ and $433 \mathrm{~K}$. A clearly separated, sharp melting endotherm can be observed independent of the heating rate at $447 \mathrm{~K}$ in this sample indicating a melt of a fraction of the particles (Figure 6). In other samples no melting and recrystallization is observed but the conversion takes place solely by solid-solid conversion (Figure 7, Figure 4).

\subsection{Temperature dependent terahertz spectroscopy of sulfathiazole - During} the TPS measurements the temperature in the centre of the sample cannot be determined with the same accuracy as for the DSC measurements. The spectra are recorded in transmission thus the thermocouple of the temperature controller needs to be placed very near to the sample rather than on the sample directly to measure the temperature of the heating element. This may lead to an underestimation of the temperature at the centre of the sample. However, this is a systematic error as sample weight and size as well as heating or cooling rate all remain constant for the different sample pellets. Consistent with this the measurements are reproducible.

PTFE used for diluting the sample material during the temperature dependent terahertz measurements exhibit spectral features that increase in intensity at higher temperatures. This is due to an annealing process and crystallization of PTFE upon heating the material below its melting temperature of about $594 \mathrm{~K}^{30,31}$ The amount of PTFE in the sample and reference pellets were the same so no effects of the PTFE spectrum on the terahertz spectra of the sulfathiazole samples should be observed. However, the spectral range for the heating experiments of sulfathiazole I to IV (Figure 8A-D) was restricted to frequencies below $90 \mathrm{~cm}^{-1}$ as subtle spectral signatures 
originating from the PTFE could not be avoided in all samples. Sample and reference were not measured at the same time but in two consecutive runs as the heating jacket only allowed the accommodation of only one sample at a time. In form $\mathrm{V}$ all the spectral features of PTFE could be compensated by the reference and for this sample the spectral range up to $120 \mathrm{~cm}^{-1}$ is shown (Figure 8E).

As anticipated from the small number of spectral features in form I no dramatic changes are observed in the terahertz spectrum during heating (Figure 8A). However, the effect of the elevated temperature on the position and intensity of the principal peak is quite distinct. At room temperature the major peak in the terahertz spectrum of form I exhibits a maximum at $65 \mathrm{~cm}^{-1}$. This peak red-shifts to $58 \mathrm{~cm}^{-1}$ at $463 \mathrm{~K}$. Its intensity decreases by $40 \%$ and a general broadening of the peak shape can be observed. These effects are rather pronounced compared to the spectral response of the other forms upon heating. Again, the very high anisotropic lattice expansion is probably responsible for these findings. ${ }^{1}$ None of the features at $37 \mathrm{~cm}^{-1}, 43 \mathrm{~cm}^{-1}, 53 \mathrm{~cm}^{-1}$, and $80 \mathrm{~cm}^{-1}$ can be distinguished from the main spectral feature at $463 \mathrm{~K}$. Upon cooling the sample back to room temperature, all peaks re-emerge and shift to their initial position (Figure 8A).

The DSC analysis of the sample of sulfathiazole form II revealed that the polymorphic form was not pure (Figure 4). Even though form II could be crystallised in its pure form (confirmed by FT-IR) the samples were extremely unstable and partially recrystallized to form III, IV or V between manufacture and the TPS analysis. Several unsuccessful attempts were made in order to obtain a stable pure specimen. As it was not possible to crystallise form II at the site of TPS analysis, the sample with the highest polymorphic purity, as determined by DSC, was chosen for the discussion of the phase transition of form II. All spectral features red-shift and decrease in intensity 
upon heating (Figure $8 \mathrm{~B}$ ). The strongest decrease in intensity can be observed for the peak at $38 \mathrm{~cm}^{-1}$. At $403 \mathrm{~K}$ an additional peak at $21 \mathrm{~cm}^{-1}$ starts to build up whereas the peak at $38 \mathrm{~cm}^{-1}$ can no longer be fully resolved. It is only a shoulder on the $33 \mathrm{~cm}^{-1}$ peak and further heating leads to the disappearance of this feature. In contrast the shoulder at $28 \mathrm{~cm}^{-1}$ is increasing in intensity and from $423 \mathrm{~K}$ onwards starts to dominate the three spectral features at low wave numbers. The effects that can be observed at $403 \mathrm{~K}$ are due to the conversion of the recrystallized impurity of form III, IV or $\mathrm{V}$ to form I in the sample of form II. At temperatures above $423 \mathrm{~K}$ the conversion is complete and the spectra represent a mixture of form II and I. The peak at $61 \mathrm{~cm}^{-1}$ at room temperature in the sample of form II shifts to lower frequencies and is broadened upon heating. At $463 \mathrm{~K}$ two local maxima can be distinguished on this feature at $53 \mathrm{~cm}^{-1}$ and $61 \mathrm{~cm}^{-1}$. From $473 \mathrm{~K}$ onwards all remaining spectral features diminish, indicating the melt of form II and only diffuse absorption can be observed at $483 \mathrm{~K}$. Cooling of the sample results in spectra as recorded for form I (Figure 8A).

In order to study the conversion process in samples of polymorphic mixtures in more detail a sample of sulfathiazole form II with a higher content of recrystallized form III, IV or V was heated to $463 \mathrm{~K}$, just below the melting point of form II. In agreement with the DSC data (Figure 9), two changes in the spectra of heated form II can be observed by TPS. Initially the spectral features red-shift and decrease in intensity. At temperatures above $413 \mathrm{~K}$ an additional peak emerges at $20 \mathrm{~cm}^{-1}$. The peaks at $26 \mathrm{~cm}^{-1}$ and $31 \mathrm{~cm}^{-1}$ change their relative intensities at $443 \mathrm{~K}$ and the features at $43 \mathrm{~cm}^{-1}$ and $48 \mathrm{~cm}^{-1}$ disappear. This indicates the solid-solid conversion of the form III/IV/V contamination to form I. Upon further heating to $463 \mathrm{~K}$ the spectrum changes again with the features at $26 \mathrm{~cm}^{-1}$ and $31 \mathrm{~cm}^{-1}$ changing intensities (Figure 10A). At 
$463 \mathrm{~K}$ the spectrum of form II is superimposed by the spectrum of form I. Cooling this sample leads to a blue-shift and sharpening of the features observed at $463 \mathrm{~K}$ (Figure 10B). The mixture of form I and II does not show any phase transitions upon cooling to room temperature.

The energy barrier to conversion of the three higher melting polymorphs (III, IV and $\mathrm{V}$, all of which melt at $447 \mathrm{~K}$ to $450 \mathrm{~K}$ ) to polymorph $\mathrm{I}$ is extremely high. Transition occurs via defect structures, as can be readily seen during hot stage microscopy. Consequently, the detailed temperature behaviour varies from crystal to crystal and sample to sample, although the overall pattern is similar.

Heating form III from room temperature results in red-shift and decreasing intensity of the spectral features as in the case of forms I and II (Figure 8C). For this sample the beginning of the polymorphic transition to form I is observed above $433 \mathrm{~K}$. Starting with a decrease in intensity of the peak at $45 \mathrm{~cm}^{-1}$ and $50 \mathrm{~cm}^{-1}$ as well as the shoulder at $56 \mathrm{~cm}^{-1}$ the peaks of form III disappear. At $463 \mathrm{~K}$ a complete conversion can be observed and only form I features can be recorded. As the sample is cooled to room temperature a blue-shift of the form I spectral features similar to that in Figure 8A takes place.

In this sample of form IV the spectral changes that indicate a change in polymorphic form during the heating process start at lower temperatures than in form III (Figure 8D). This behaviour corresponds very well to the DSC trace. At $403 \mathrm{~K}$ the intensity of the peak located at $82 \mathrm{~cm}^{-1}$ at room temperature decreases by $15 \%$ compared to the intensity at $393 \mathrm{~K}$. In parallel between $25 \mathrm{~cm}^{-1}$ and $70 \mathrm{~cm}^{-1}$ a very broad spectral feature, characteristic of form I, increases in absorption. Its intensity is 
1.36 times higher at $403 \mathrm{~K}$ compared to $393 \mathrm{~K}$ and 1.58 times increased at $413 \mathrm{~K}$ compared to $393 \mathrm{~K}$. In the spectrum at $423 \mathrm{~K}$ no form IV features are detectable. Lower wavenumber features of form IV can no longer be discerned. Further heating to $473 \mathrm{~K}$ of the converted form I leads to a spectrum similar to Figure $8 \mathrm{~A}$ only showing bands of sulfathiazole form I. It is interesting to note that, prior to the conversion from form IV to I, the peaks of form IV show substantial red-shift with heating. The peak positions shift between $2 \mathrm{~cm}^{-1}$ and $6 \mathrm{~cm}^{-1}$ with the peak at $82 \mathrm{~cm}^{-1}$ showing the largest shift to $76 \mathrm{~cm}^{-1}$.

As for forms III and IV, conversion to form I can be followed upon heating the sample of form V. Initially, the intensity ratio of the peaks at $33 \mathrm{~cm}^{-1}$ and $38 \mathrm{~cm}^{-1}$ reverses. At room temperature the peak at $38 \mathrm{~cm}^{-1}$ has a slightly higher intensity than the peak at $33 \mathrm{~cm}^{-1}$. An increase in temperature leads to a decrease in intensity of the $38 \mathrm{~cm}^{-1}$ peak and a red-shift of its position. In contrast the peak at $33 \mathrm{~cm}^{-1}$ also redshifts but the decrease in intensity is much smaller. The temperature where the intensity of both peaks is the same varies for samples of different crystallisation batches. In one sample it was found to be at $363 \mathrm{~K}$ whereas in another sample it was at $423 \mathrm{~K}$. The peaks at $62 \mathrm{~cm}^{-1}$ and $113 \mathrm{~cm}^{-1}$ both steadily decrease in intensity. From $433 \mathrm{~K}$ onwards the form I spectrum starts to dominate (Figure $8 \mathrm{E}$ ) and at $463 \mathrm{~K}$ only features of form I can be detected in the spectrum of the sample. Again this corresponds well to the observations recorded by DSC.

\section{Conclusion}

We have demonstrated that by using TPS the five known polymorphic forms of sulfathiazole can be unequivocally discriminated from one another. Even though the 
spectral features cannot yet be assigned to structural properties, the potential of spectroscopy in the terahertz region for the characterisation of polymorphic forms is evident. Furthermore the information that can be extracted from temperature dependent measurements was highlighted and utilised for additional characterisation of this polymorphic system. The temperature induced phase transitions in all the different forms of sulfathiazole were studied in detail and compared with the data from thermal analysis.

\section{Acknowledgements}

The authors would like to acknowledge Fang Tian, University of Otago for acquiring the hot stage polarising light microscopy images and Paul Gabbott, PerkinElmer UK for helpful assistance with the high speed DSC measurements.

\section{Figure legends}

Figure 1: Chemical structure of sulfathiazole.

Figure 2: A) Terahertz absorption spectra and B) Low frequency Raman spectra for the different polymorphic forms of sulfathiazole. For clarity spectra are offset in absorbance and normalized.

Figure 3: Raman spectra between $100 \mathrm{~cm}^{-1}$ and $600 \mathrm{~cm}^{-1}$ for the different polymorphic forms of sulfathiazole. For clarity spectra are offset in absorbance and normalized. 
Figure 4: DSC traces for the different forms of sulfathiazole at different heating rates. A) $2 \mathrm{~K} \mathrm{~min}^{-1}$ B) $\left.10 \mathrm{~K} \mathrm{~min}^{-1} \mathrm{C}\right) 150 \mathrm{~K} \mathrm{~min}^{-1}$ D) $300 \mathrm{~K} \mathrm{~min}^{-1}$ E) $500 \mathrm{~K} \mathrm{~min}^{-1}$. A-C heat flux DSC; D-E power compensation DSC.

Figure 5: Conversion of sulfathiazole form IV to form I studied by polarizing light microscopy. In this sample batch part of the sample convert to form I by melt and recrystallization whereas the bigger particles convert via a solid-solid transition.

Figure 6: Sulfathiazole form IV. DSC traces for a sample of form IV recorded at different heating rates. The sample contains a fraction of particles converting to form I via solid-solid transition and the rest of the particles melting at the melting point of form IV. A) $\left.2 \mathrm{~K} \mathrm{~min}^{-1} \mathrm{~B}\right) 10 \mathrm{~K} \mathrm{~min}^{-1} \mathrm{C}$ ) $150 \mathrm{~K} \mathrm{~min}^{-1}$.

Figure 7: Conversion of sulfathiazole form IV to form I studied by polarizing light microscopy. Here all particles convert to form I via a solid-solid transition.

Figure 8: Sulfathiazole forms I, II, III, IV and V. A) Form I heating from 293 K to $463 \mathrm{~K}$ and subsequent cooling back to room temperature. Heating from room temperature to $473 \mathrm{~K}$ : B) form II. C) form III; D) form IV, and E) form V.

Figure 9: Impure sulfathiazole form II. DSC trace for a sample of form II that has partly recrystallized to form III, IV or $\mathrm{V}$ recorded at different heating rates. A) $\left.\left.2 \mathrm{~K} \mathrm{~min}^{-1} \mathrm{~B}\right) 10 \mathrm{~K} \mathrm{~min}^{-1} \mathrm{C}\right) 150 \mathrm{~K} \mathrm{~min}^{-1}$. 
Figure 10: Sulfathiazole form II that has partly recrystallized to form III, IV or V. A) Heating from room temperature to $463 \mathrm{~K}$. B) Cooling back to room temperature.

\section{Figures}<smiles>Nc1ccc(S(=O)(=O)/N=c2/[nH]ccs2)cc1</smiles>

Figure 1 

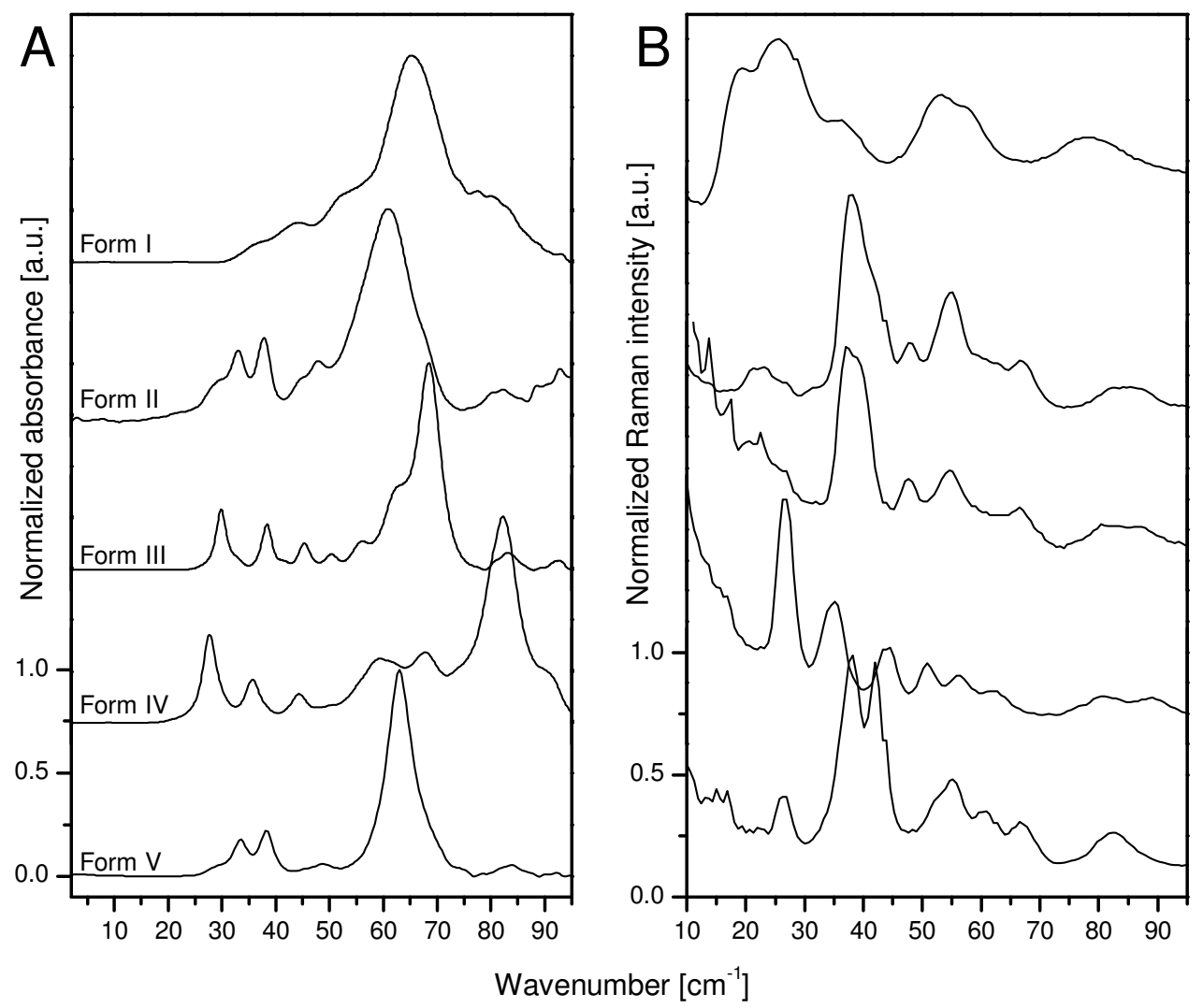

Figure 2 


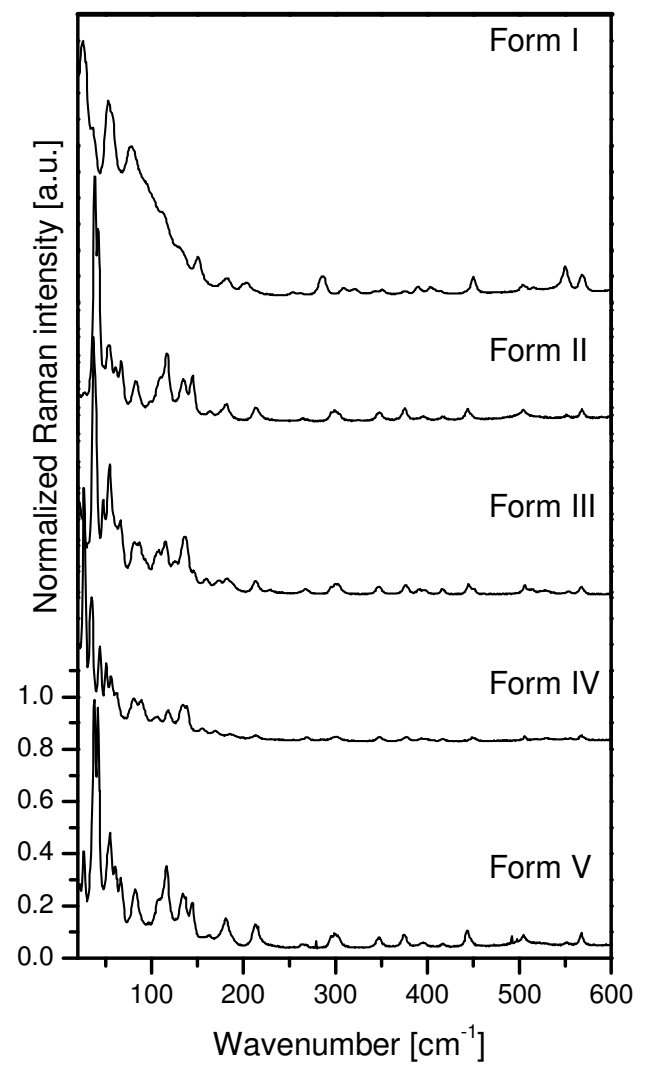

Figure 3 


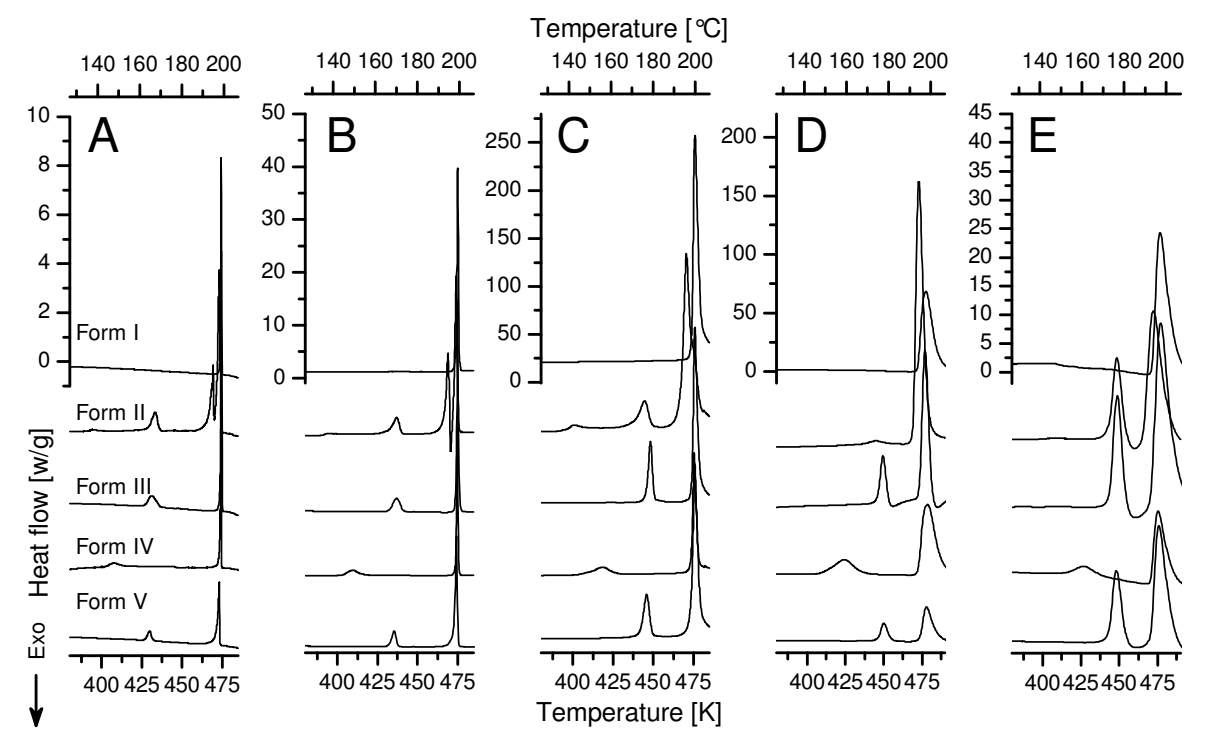

\section{Figure 4}

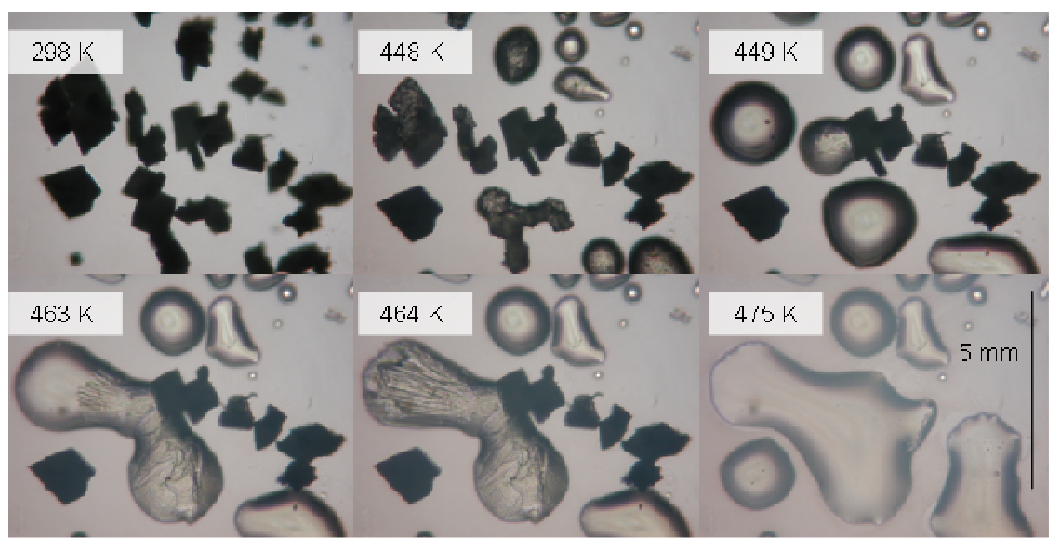

Figure 5 


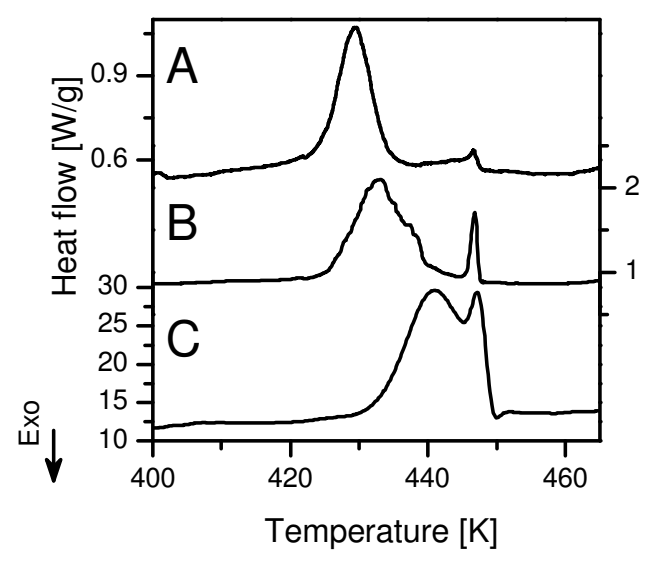

Figure 6

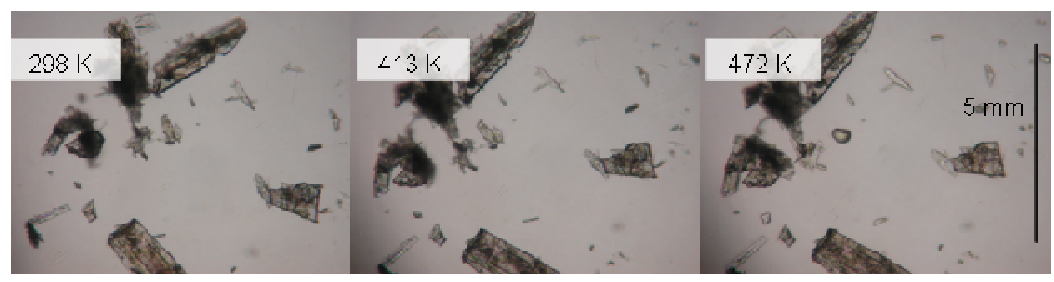

Figure 7 

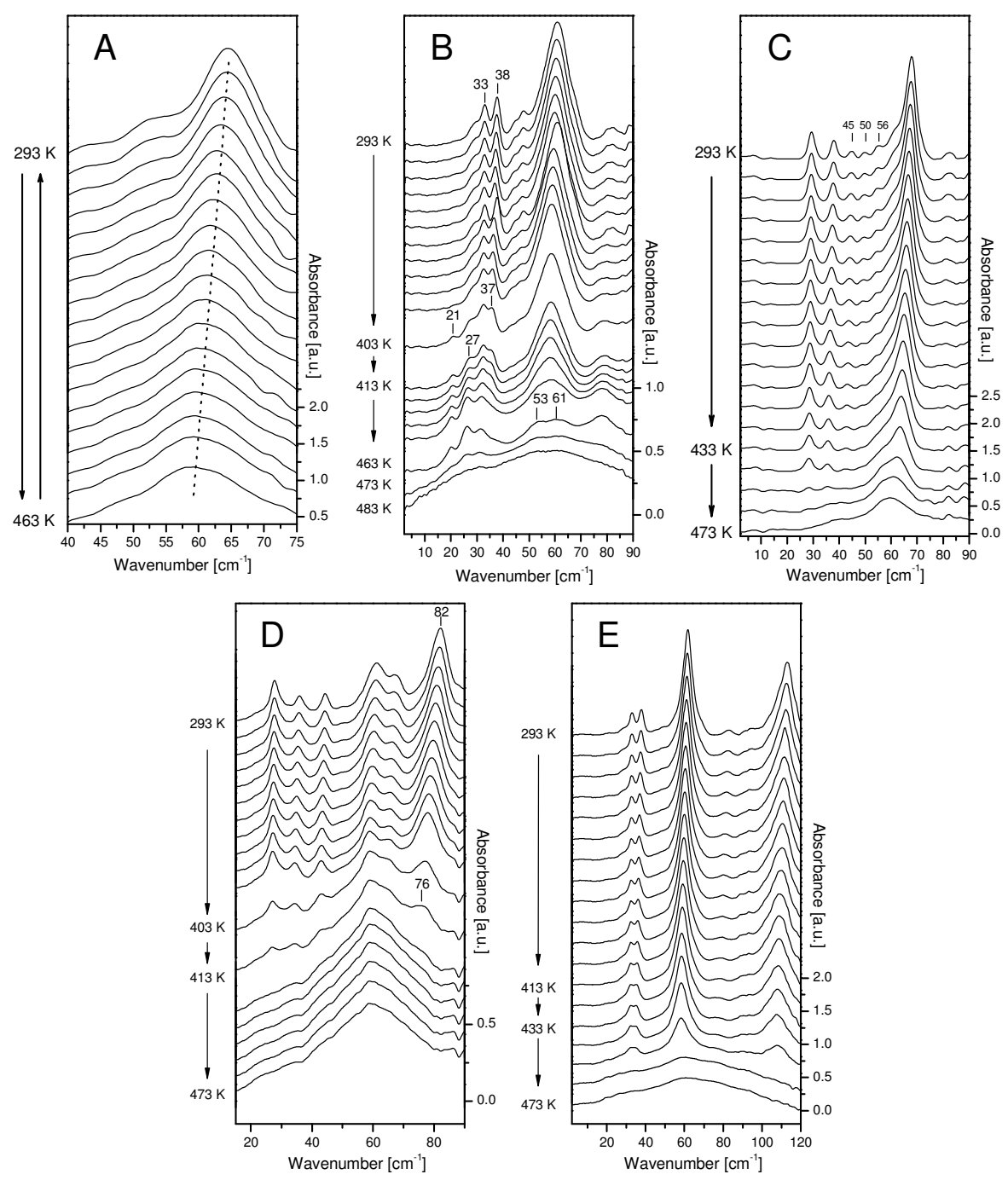

Figure 8 


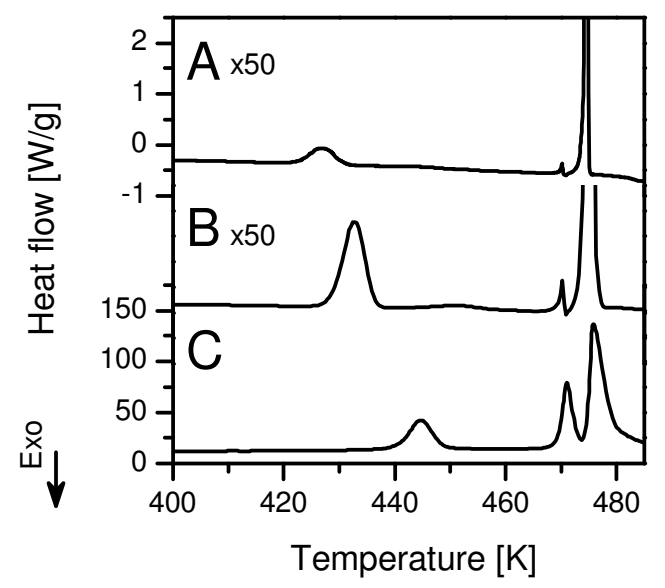

Figure 9

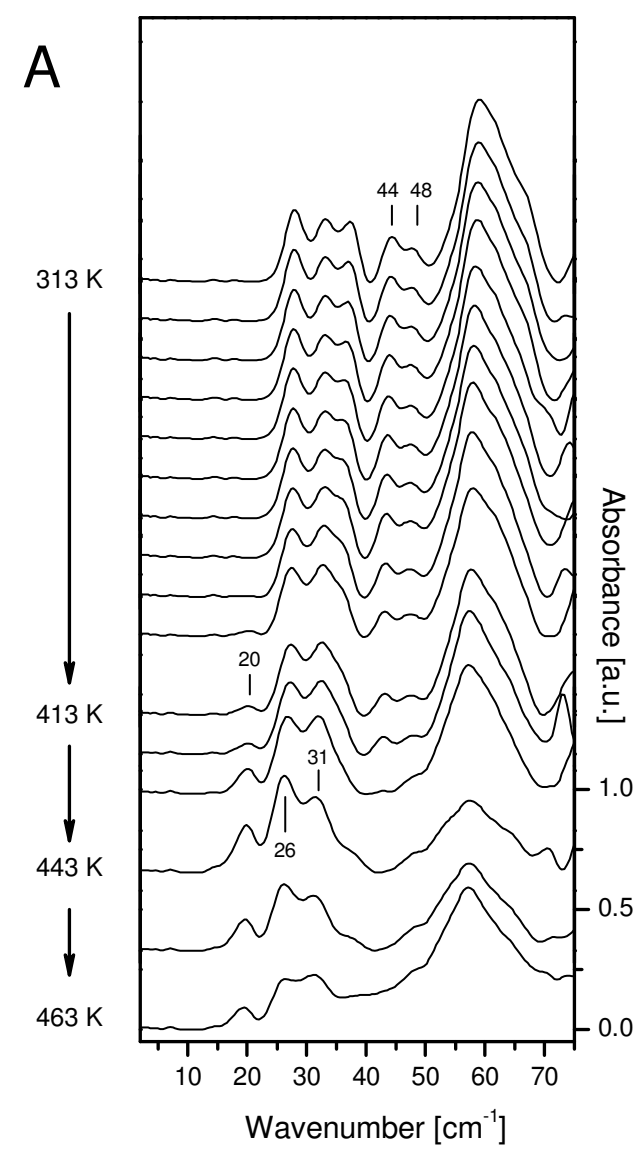

B

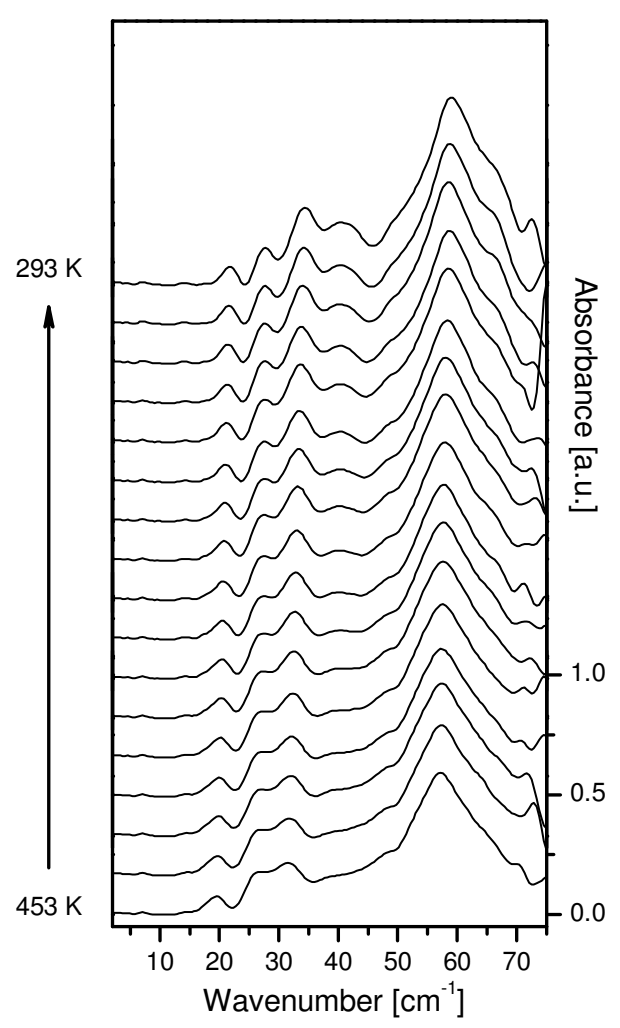

Figure 10 


\section{Table captions}

Table 1: Positions of the far-infrared spectral features of sulfathiazole polymorphic forms. (w) Weak spectral feature; (s) shoulder. Brackets indicate unresolved peaks.

Table 2: Melting point and heat of fusion data from the DSC experiments at different heating rates. $2 \mathrm{~K} \mathrm{~min}^{-1}, 10 \mathrm{~K} \mathrm{~min}^{-1}$ and $150 \mathrm{~K} \mathrm{~min}^{-1}$ recorded on a heat flux setup; $300 \mathrm{~K} \mathrm{~min}^{-1}$ and $500 \mathrm{~K} \mathrm{~min}^{-1}$ recorded on a power compensation setup. ${ }^{\text {a) }}$ The two peaks are not fully resolved. ${ }^{\text {b) }}$ The heat of fusion represents form II and the impurity of form I together. Peaks were not resolved.

\section{Tables}

\begin{tabular}{lll}
\hline Polymorphic form & IR active modes & Raman active modes \\
\hline Form I & $37_{\mathrm{w}}, 43_{\mathrm{w}}, 53_{\mathrm{w}}, 65,80_{\mathrm{w}}$ & $\left(20,27,39_{\mathrm{w}}\right),(54,60),\left(80,98_{\mathrm{w}}\right)$ \\
Form II & $\left(28_{\mathrm{s}}, 33,38\right),\left(44_{\mathrm{s}}, 48\right), 61,82$ & $(39,42), 54_{\mathrm{w}}, 62,67,83$ \\
Form III & $\left(30,33_{\mathrm{w}}\right),\left(38,41_{\mathrm{w}}\right), 45,50,56_{\mathrm{s}}$, & $37,48,55,66,\left(81_{\mathrm{w}}, 87_{\mathrm{w}}\right)$ \\
& $(62,68), 83,92$ & \\
Form IV & $28,36,44,(60,68),\left(82,90_{\mathrm{w}}\right)$ & $28,36,44,51,56_{\mathrm{w}}, 63_{\mathrm{w}},\left(80_{\mathrm{w}}, 90_{\mathrm{w}}\right)$ \\
Form V & $\left(28_{\mathrm{w}}, 33,38\right),\left(45_{\mathrm{w}}, 48_{\mathrm{w}}\right), 63,84$ & $26,(38,42), 55,61_{\mathrm{w}}, 67_{\mathrm{w}}, 82_{\mathrm{w}}$ \\
\hline \multicolumn{1}{c}{ Table 1 } & &
\end{tabular}

\begin{tabular}{|c|c|c|c|c|c|c|}
\hline \multirow[b]{2}{*}{$\begin{array}{l}\text { Polymorphic } \\
\text { form }\end{array}$} & \multicolumn{5}{|c|}{ Melting point (onset $\mathrm{K})$ / Heat of fusion $\left(\mathrm{J} \mathrm{g}^{-1}\right)$ at different heating rates } & \\
\hline & $2 \mathrm{~K} \mathrm{~min}^{-1}$ & $10 \mathrm{~K} \mathrm{~min}^{-1}$ & $150 \mathrm{~K} \mathrm{~min}^{-1}$ & $300 \mathrm{~K} \mathrm{~min}^{-1}$ & $500 \mathrm{~K} \mathrm{~min}^{-1}$ & \\
\hline Form I & $474.1 / 108.1$ & $474.7 / 81.8$ & $474.4 / 205.5$ & $472.4 / 106.2$ & $471.6 / 99.6$ & \\
\hline \multirow[t]{2}{*}{ Form II } & $423.2 / 26.2$ & $428.8 / 24.9$ & $440.8 / 38.4$ & $442.8 / 17.2$ & 442.9 / 32.9 & \\
\hline & $\begin{array}{ll}469.9 / & 1.2 \\
474.1 / 115.1\end{array}$ & $\begin{array}{l}469.6 / r 1.0 \\
474.2 / 115.5\end{array}$ & $\begin{array}{l}469.6 / 34.2^{\mathrm{a})} \\
474.8 / 168.2^{\mathrm{a}}\end{array}$ & 468.3 / 82.4 & $466.2 / 75.4^{\mathrm{b})}$ & \multirow{2}{*}{$\begin{array}{l}\text { Comment [AZ1]: We need to } \\
\text { update this value before } \\
\text { submission. I have mailed Paul } \\
\text { Gabbot in order to get it. }\end{array}$} \\
\hline \multirow[t]{2}{*}{ Form III } & 426.9 / 30.1 & $432.4 / 27.3$ & 445.8 / 49.34 & $446.0 / 45.1$ & $443.7 / 34.5$ & \\
\hline & $474.1 / 110.8$ & $474.1 / 114.2$ & $474.4 / 193.2$ & $473.4 / 142.0$ & $470.9 / 95.7$ & \\
\hline \multirow[t]{2}{*}{ Form IV } & $419.6 / 31.8$ & $411.0 / 32.4$ & $415.8 / 45.91$ & $410.9 / 29.7$ & $415.0 / 22.6$ & \\
\hline & $473.5 / 117.3$ & $473.6 / 116.4$ & $473.7 / 201.7$ & 472.4 / 89.81 & $470.6 / 97.34$ & \\
\hline \multirow[t]{2}{*}{ Form V } & $427.3 / 27.6$ & $432.5 / 29.5$ & $442.1 / 43.92$ & $446.3 / 29.8$ & $443.5 / 34.3$ & \\
\hline & $471.7 / 112.6$ & $472.9 / 114.7$ & $473.5 / 202.3$ & $473.7 / 80.6$ & $470.5 / 79.3$ & \\
\hline
\end{tabular}




\section{References}

1. Apperley DC, Fletton RA, Harris RK, Lancaster RW, Tavener S, Threlfall TL 1999. Sulfathiazole polymorphism studied by magic-angle spinning NMR. J Pharm Sci 88(12):1275-1280.

2. Burger A, Dialer RD 1983. New Study Results on the Polymorphism of Sulfathiazol. Pharmaceutica Acta Helvetiae 58(3):72-78.

3. Hughes DS, Hursthouse MB, Lancaster RW, Tavener S, Threlfall T, Turner P 1997. How many polymorphs has sulfathiazole? Proposals for reporting crystallographic data of polymorphs. J Pharm Pharmacol 49(S4):20.

4. Hughes DS, Hursthouse MB, Threlfall T, Tavener S 1999. A new polymorph of sulfathiazole. Acta Crystallographica Section C 55(11):1831-1833.

5. Chan FC, Anwar J, Cernik R, Barnes P, Wilson RM 1999. Ab initio structure determination of sulfathiazole polymorph $\mathrm{V}$ from synchrotron $\mathrm{X}$-ray powder diffraction data. Journal of Applied Crystallography 32:436-441.

6. Lagas M, Lerk CF 1981. The Polymorphism of Sulfathiazole. Int J Pharm $8(1): 11-24$.

7. Anwar J, Tarling SE, Barnes P 1989. Polymorphism of Sulfathiazole. J Pharm Sci 78(4):337-342.

8. Blagden N, Davey RJ, Lieberman HF, Williams L, Payne R, Roberts R, Rowe R, Docherty R 1998. Crystal chemistry and solvent effects in polymorphic systems - Sulfathiazole. J Chem Soc-Faraday Trans 94(8):1035-1044.

9. Bingham AL, Hughes DS, Hursthouse MB, Lancaster RW, Tavener S, Threlfall TL 2001. Over one hundred solvates of sulfathiazole. Chem Commun (7):603604.

10. Blagden N, Davey RJ, Rowe R, Roberts R 1998. Disappearing polymorphs and the role of reaction by-products: the case of sulphathiazole. Int J Pharm 172(12):169-177.

11. Day GM, Zeitler JA, Jones W, Rades T, Taday PF 2005. Understanding the influence of polymorphism on phonon spectra: lattice dynamics calculations and terahertz spectroscopy of carbamazepine. J Phys Chem B in press. 
12. Taday PF, Newnham DA 2004. Technological advances in terahertz pulsed systems bring far-infrared spectroscopy into the spotlight. Spectroscopy Europe 16(5):20-24.

13. Dorney TD, Baraniuk RG, Mittleman DM 2001. Material parameter estimation with terahertz time-domain spectroscopy. J Opt Soc Am A-Opt Image Sci Vis 18(7):1562-1571.

14. Ferguson B, Zhang XC 2002. Materials for terahertz science and technology. Nat Mater 1(1):26-33.

15. Beard MC, Turner GM, Schmuttenmaer CA 2002. Terahertz spectroscopy. J Phys Chem B 106(29):7146-7159.

16. Walther M, Fischer B, Schall M, Helm H, Jepsen PU 2000. Far-infrared vibrational spectra of all-trans, 9-cis and 13-cis retinal measured by THz timedomain spectroscopy. Chem Phys Lett 332(3-4):389-395.

17. Shen YC, Upadhya PC, Linfield EH, Davies AG 2003. Temperature-dependent low-frequency vibrational spectra of purine and adenine. Appl Phys Lett 82(14):2350-2352.

18. Taday PF, Bradley IV, Arnone DD, Pepper M 2003. Using terahertz pulse spectroscopy to study the crystalline structure of a drug: A case study of the polymorphs of ranitidine hydrochloride. J Pharm Sci 92(4):831-838.

19. Walther M, Fischer BM, Jepsen PU 2003. Noncovalent intermolecular forces in polycrystalline and amorphous saccharides in the far infrared. Chem Phys 288(2-3):261-268.

20. Watanabe Y, Kawase K, Ikari T, Ito H, Ishikawa Y, Minamide H 2003. Component spatial pattern analysis of chemicals using terahertz spectroscopic imaging. Appl Phys Lett 83(4):800-802.

21. Kutteruf MR, Brown CM, Iwaki LK, Campbell MB, Korter TM, Heilweil EJ 2003. Terahertz spectroscopy of short-chain polypeptides. Chem Phys Lett 375(3-4):337-343.

22. Strachan CJ, Rades T, Newnham DA, Gordon KC, Pepper M, Taday PF 2004. Using terahertz pulsed spectroscopy to study crystallinity of pharmaceutical materials. Chem Phys Lett 390(1-3):20-24. 
23. Strachan CJ, Taday PF, Newnham DA, Gordon KC, Zeitler JA, Pepper M, Rades T 2005. Using terahertz pulsed spectroscopy to quantify pharmaceutical polymorphism and crystallinity. J Pharm Sci 94(4):837-846.

24. Zeitler JA, Newnham DA, Taday PF, Strachan CJ, Pepper M, Gordon KC, Rades T 2005. Temperature dependent terahertz pulsed spectroscopy of carbamazepine. Thermochim Acta 436(1-2):71-77.

25. Berg RW, Kerridge DH 2004. The NaNO3/KNO3 system: the position of the solidus and sub-solidus. Dalton Transactions (15):2224-2229.

26. Threlfall T, Southampton, UK, Personal communication with Zeitler JA. 2005

27. Burger A, Ramberger R 1979. On the Polymorphism of Pharmaceuticals and Other Molecular Crystals. II. Mikrochim Acta II:273-316.

28. Boldyreva E, Novosibirsk Academy of Sciences, Personal communication with Threlfall T. 2003

29. McGregor C, Saunders MH, Buckton G, Saklatvala RD 2004. The use of highspeed differential scanning calorimetry (hyper-DSC) to study the thermal properties of carbamazepine polymorphs. Thermochim Acta 417(2):231-237.

30. Chantry GW, Fleming JW, Nicol EA, Willis HA, Cudby MEA, Boerio FJ 1974. Far Infrared-Spectrum of Crystalline Polytetrafluoroethylene. Polymer 15(2):69-73.

31. Willis HA, Cudby MEA, Chantry GW, Nicol EA, Fleming JW 1975. Far Infrared Study of Conformational Disorder in Ptfe. Polymer 16(2):74-76. 